\title{
The importance of high-index surfaces for the morphology of GaAs quantum dots
}

\author{
J. Platen, A. Kley, C. Setzer, K. Jacobi, ${ }^{a)}$ P. Ruggerone, ${ }^{\text {b) }}$ and M. Scheffler \\ Fritz-Haber-Institut der Max-Planck-Gesellschaft, Faradayweg 4-6, 14195 Berlin, Germany
}

(Received 24 July 1998; accepted for publication 29 December 1998)

\begin{abstract}
Results of a combined experimental and theoretical study are presented. GaAs $\{113\}$ and $\{112\}$ surfaces have been prepared by molecular beam epitaxy and analyzed in situ by low-energy electron diffraction and ex situ by atomic-force microscopy. The experimentally found structures are in full agreement with surface energies calculated by density-functional theory. The $\{112\}$ surfaces are unstable under faceting into low-index planes, whereas the $\{113\}$ surfaces appear as singular surfaces. Particularly, for GaAs(113) the surface energy is comparable with the values for the low-index surfaces. The impacts of these results on the interface between InAs quantum dots and the embedding GaAs matrix are discussed. (C) 1999 American Institute of Physics.

[S0021-8979(99)02907-2]
\end{abstract}

\section{INTRODUCTION}

The reduction of the size of a semiconductor crystal to several dozens of interatomic distances produces a significant change in the main properties of the material because of the new size-dependent quantizations. The ultimate limit is represented by quantum dots, that is, semiconductor structures characterized by geometrical confinement in all three dimensions. For these systems the deviation from the bulk properties is particularly strong. The technological impact of these structures appears relevant for the realization of devices like, for example, diode lasers with quantum dots as active media: increased material gain, reduced threshold current density, higher frequencies of operation, and high control over the emission spectrum were predicted for the devices based on quantum dots. ${ }^{1}$ These predictions were recently confirmed ${ }^{2}$ and have inspired several studies. ${ }^{2-10}$ To achieve these results strict requirements on dot size, shape, uniformity, and density had to be satisfied, and the open question regards the preparation of a system with the required characteristics.

Quantum dots can be prepared by embedding small three-dimensional clusters of low-band gap material in a wide-band gap semiconductor matrix. However, to realize a system characterized by single bound states the size of the quantum dot should lie within a specific range determined by the electronic de Broglie wavelength. Quantum dots must be not too large, otherwise the separation between energy levels becomes comparable to the thermal energy and the population of higher-lying levels cannot be ignored. For InAs quantum dots in GaAs the diameter was estimated to lie between 4 and $20 \mathrm{~nm} .{ }^{2}$ Moreover, for optical applications dislocations and defects should be absent in the quantum dots and in the regions in their vicinity. Finally, dense $\left(\sim 10^{11} \mathrm{~cm}^{-2}\right)$ and ordered arrays of quantum dots with a very narrow size and

\footnotetext{
${ }^{\text {a)} C o r r e s p o n d i n g ~ a u t h o r ; ~ E l e c t r o n i c ~ m a i l: ~ j a c o b i @ f h i-b e r l i n . m p g . d e ~}$

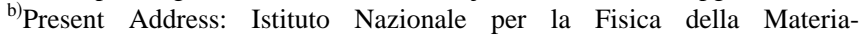
Dipartimento di Fisica, Universitá di Cagliari, Italy.
}

shape distribution are required for most of the applications.

Such small structures cannot be prepared by standard lithography techniques. Instead strain-induced self-assembly of small islands has been successfully used to create, e.g., small InAs islands within a GaAs matrix grown on a $\operatorname{GaAs}(100)$ surface with a rather narrow size distribution besides very similar shape and orientation. ${ }^{4-10}$ The current understanding of this process is the following: Since the lattice mismatch between InAs and GaAs amounts to 7\%, only the first 1.5 monolayers of InAs grow pseudomorphic on, e.g., GaAs(100). For larger thicknesses the InAs layer coagulates into small three-dimensional islands in order to minimize the sum of elastic energy, interface, and surface energies. ${ }^{3}$ So far theoretical studies have mainly taken into account only lowindex surfaces as possible delimiting faces of the quantum dots. For instance, based on this assumption, the equilibrium structure of uncovered InAs islands are calculated to be hills bounded by $\{110\},\{111\}$, and $\{\overline{111}\}$ facets and the (001) surface on top. Although this hypothesis is very reasonable as first approximation, the occurrence of high-index planes should not be discarded. An experimental assessment on the actual shape of the dot is difficult to achieve, and only for InP dots atomic force (AFM) and transmission electron microscopy (TEM) studies exist, ${ }^{11}$ confirming the theoretical prediction. ${ }^{3}$ For InAs quantum dots the situation is less clear, so far. For example, from the transmission electron micrographs it is not possible so far to uncover whether a pyramidally shaped cluster is flattened or not and whether or not the edges are rounded by high-index surfaces. Clearly, a careful control on the characteristics of the assembled arrays of quantum dots as well as of the single quantum dot should be a primary aim of basic and applied research. Moreover, the need of a possibly controlled size distribution and density raises the important question on adequate substrates that can serve as template for the assembling of the quantum dots.

Despite the relevance, a clear cut picture of the structural characteristics of the high-index GaAs surfaces in their role of limiting faces of and possible substrates for quantum dots is still missing. The complexity of the occurring structures 
The ideal truncated surfaces

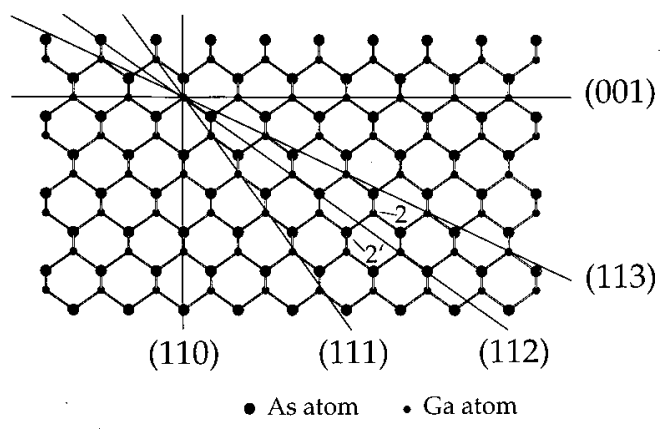

FIG. 1. Ball-and-stick model of ideal, bulk truncated GaAs cut along the (110) plane. The lines where the (001), (113), (112), (111), and (110) plane cut the figure are indicated.

and the sensitivity on the chemical environments represent a challenging task for measurements and calculations. The wide range of structures and configurations that can occur under different chemical conditions points out strongly the necessity of new theoretical and experimental investigations. In the following we report such a study that combines experimental and theoretical studies of the GaAs $\{112\}$ and $\{113\}$ surfaces. Figure 1 shows a view of the atomic structure of a GaAs single crystal along the (110) plane with the labeling of the (100), (110), (111), (112), and (113) planes. We note that (112) and (113) are polar surfaces. The polarity of the $\{112\}$ surfaces derives from the nature of the atom with a broken bond in the second layer which is a $\mathrm{Ga}$ atom for (112) (marked 2 in Fig. 1) and an As atom for the (112) (marked $2^{\prime}$ in Fig. 1). On $\{112\}$ the Ga-As bond of the surface atoms is parallel to the surface, whereas this bond is tilted on $\{113\}$. Therefore, there is a $\mathrm{Ga}$ atom in the topmost layer of (113) and an As atom for (113), i.e., the (113) surfaces are polar.

\section{EXPERIMENT AND THEORY}

Experimentally, the surfaces have been analyzed with spot-profile analysis low-energy electron diffraction (SPALEED), surface core level photoemission spectroscopy (using synchrotron radiation at BESSY I, Berliner Elektronenspeichering-Gesellschaft für Synchrotronstrahlung), AFM, and scanning electron microscopy (SEM). The samples were taken from $n$-doped wafers and prepared following standard procedures known for $\mathrm{GaAs}(001)$. All preparations were carried out within a compact molecular beam epitaxy (MBE) chamber which was connected by an ultrahigh vacuum (UHV) transfer line to a UHV surface science analysis chamber. This allowed performing the analyses with LEED and surface core level shift in situ (i.e., without leaving the UHV environment whereas the microscope pictures were taken ex situ in air. Theoretically, the free surface energy has been calculated for a variety of different models for the GaAs(112) and (113) surfaces in order to determine the most stable surface morphology. These calculations are within the framework of density functional theory (DFT) us-
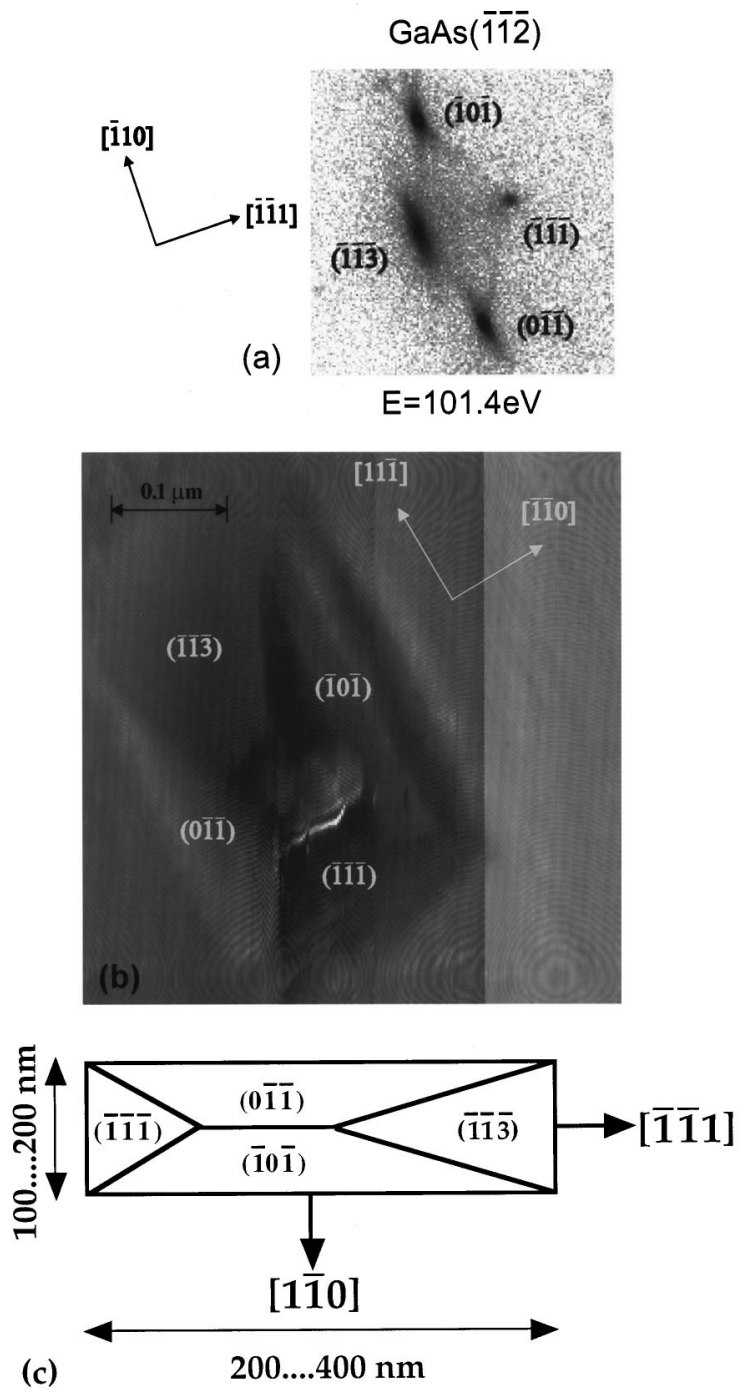

FIG. 2. (a) $(0,0)$ beam of spot profile analysis low-energy electron diffraction (SPA-LEED) pattern at a primary energy of $101.4 \mathrm{eV}$ for the GaAs (112) surface. The different facet spots are indicated. (b) Atomic force microscope image of depression into the GaAs $(\overline{112})$ surface. The terminating faces are indicated. (c) Top view of a model for the depression into the surface derived from the SPA-LEED data.

ing the local density approximation (LDA) for the exchangecorrelation functional. More details on the methods can be found elsewhere. ${ }^{12}$

\section{RESULTS AND DISCUSSION}

Examples of the experimental data are given in Fig. 2. In the upper panel [Fig. 2(a)] we show the $(0,0)$ beam from a spot-profile analysis low-energy electron diffraction (SPALEED) pattern for a MBE prepared GaAS (11/2) surface. One clearly recognizes the split into four different beams indicating strong faceting of the surface. In the middle panel [Fig. 2(b)] we present an AFM image of such a single facet. This image was taken ex situ in air. Nevertheless, the shape and the size of the facet are well observed and are in very good agreement with the result from the SPA-LEED analysis. Only the bottom (where the four different faces join together) is not clearly resolved. It cannot be said whether the 


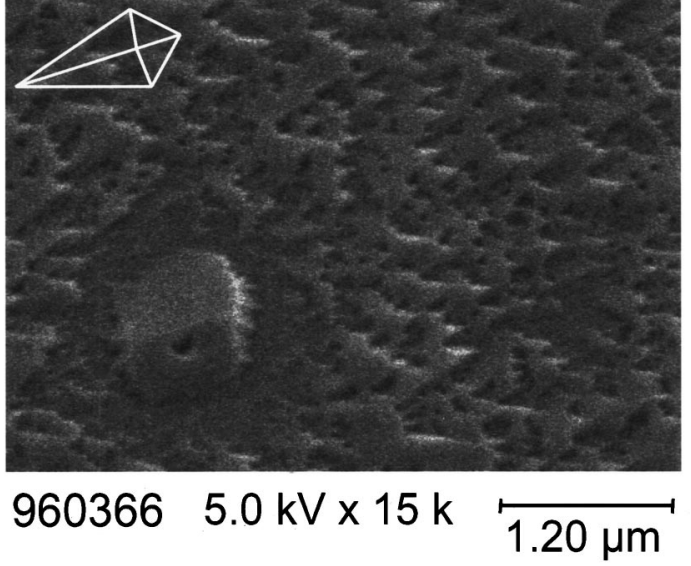

FIG. 3. Scanning electron microscope image of depressions into the GaAs (113) surface. In the inset the calculated morphology is shown.

structure is flattened there or (more likely) whether the cantilever of the AFM was not able to follow the real structure at the bottom of the facet. The morphology of these depressions was also confirmed by SEM images. In the lower panel of Fig. 2 [Fig. 2(c)] a model for a single faceted depression into the surface is depicted. Note that the diameter of the faces as derived from the width of the beams in SPA-LEED spectra is in excellent agreement with the sizes extracted from the AFM images. Moreover, all the experiments (SPA-LEED, AFM, and SEM) provide evidences that a (113) face contributes to the shape of the facet. For the (113) surface itself no superstructure has been reported in the literature. ${ }^{12}$ In our LEED experiment we observe a $1 \times 1$ structure stabilized for As-rich conditions, whereas under Ga-rich conditions the surface is not stable (see Fig. 3). From Fig. 3 pyramidal structures resulting from the faceting are recognized. Also the GaAS(112) surface is not stable but exhibits depressions with well defined faces quite similar to those observed on GaAs (112). With AFM a good resolution has not been achieved, and no assessment about the morphology can be extracted. However, SPA-LEED images seem to suggest the

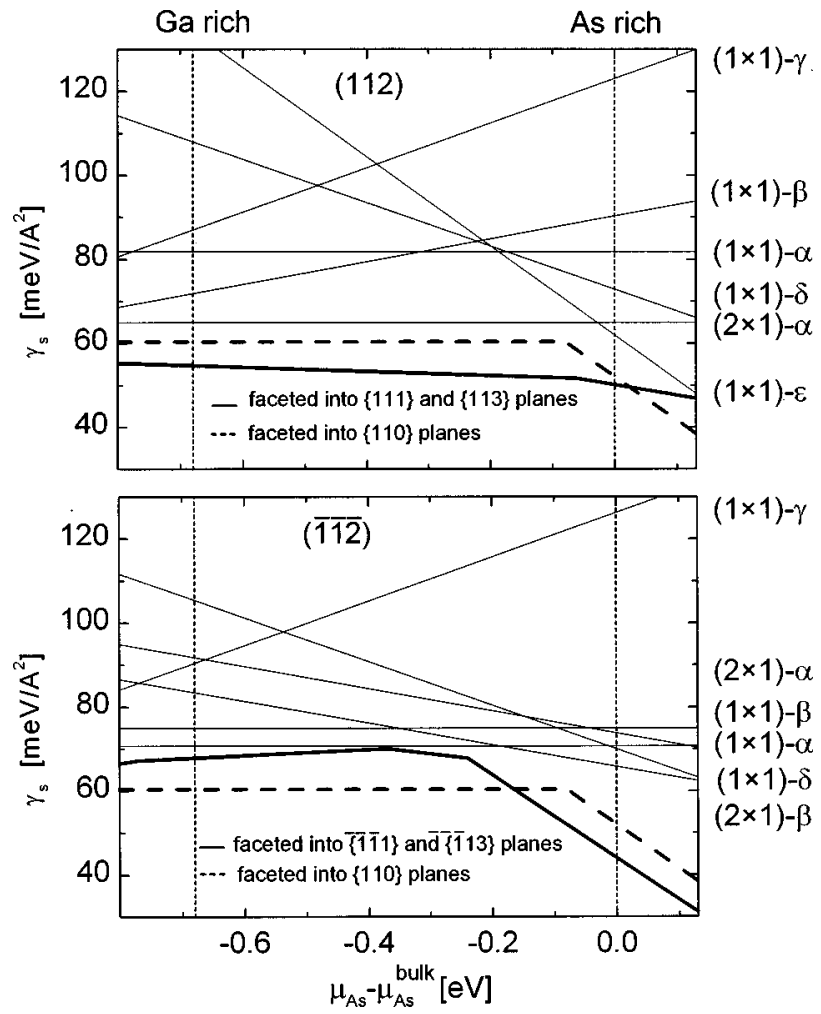

FIG. 4. Calculated surface energies of the GaAs (112) (upper part) and GaAs (11) surface (lower part) as a function of the As chemical potential. For the different models see Table I.

appearance of $\{101\},\{111\}$, and $\{124\}$ facets. For GaAs(113) our measurements confirm the absence of faceting and the appearance of an $8 \times 1$ surface reconstruction in agreement with previous experiments. ${ }^{13-15}$

In order to clarify these experimental findings we have calculated the surface energies for a number of different models listed in Table I for the $\{112\}$ and $\{113\}$ surfaces. The free surface energy $\gamma$ is defined at zero temperature as

$$
\gamma_{\text {surface }} A=E_{\text {tot }}-\mu_{\mathrm{GaAs}} N_{\mathrm{Ga}}-\mu_{\mathrm{As}}\left(N_{\mathrm{As}}-N_{\mathrm{Ga}}\right)
$$

TABLE I. Different structures for the $\{112\}$ and $\{113\}$ surfaces for which surface energies have been calculated. The starting structure is described which was relaxed in the ab initio calculations.

\begin{tabular}{|c|c|c|}
\hline Name & Surface & Starting structure \\
\hline$(1 \times 1)-\alpha$ & $\{112\}$ & truncated bulk structure \\
\hline$(1 \times 1)-\alpha$ & $(113)[(\overline{11} \overline{3})]$ & truncated bulk structure, As $[\mathrm{Ga}]$ terminated \\
\hline$(1 \times 1)-\beta$ & $(112)[(\overline{11} 2)]$ & as $(1 \times 1)-\alpha$ but 1 st layer As $[\mathrm{Ga}]$ removed \\
\hline$(1 \times 1)-\beta$ & $(113)[(\overline{113})]$ & truncated bulk structure, $\mathrm{Ga}[\mathrm{As}]$ terminated \\
\hline$(1 \times 1)-\gamma$ & $\{112\}$ & as $(1 \times 1)-\alpha$ but 1 st layer As substituted by Ga \\
\hline$(1 \times 1)-\gamma$ & $(113)[(\overline{11} \overline{3})]$ & as $(1 \times 1)-\alpha$ but 1 st layer As $[\mathrm{Ga}]$ substituted by $\mathrm{Ga}[\mathrm{As}]$ \\
\hline$(1 \times 1)-\delta$ & $\{112\}$ & as $(1 \times 1)-\alpha$ but 1 st layer Ga substituted by As \\
\hline$(1 \times 1)-\delta$ & $(113)[(\overline{113})]$ & as $(1 \times 1)-\alpha$ but 2 nd layer $\mathrm{Ga}[\mathrm{As}]$ substituted by As $[\mathrm{Ga}]$ \\
\hline$(1 \times 1)-\epsilon$ & $\{112\}$ & as $(1 \times 1)-\alpha$ but 1 st and 2 nd layer Ga substituted by As \\
\hline$(1 \times 1)-\epsilon$ & $(113)[(\overline{11} \overline{3})]$ & as $(1 \times 1)-\beta$ but 1 st $\mathrm{Ga}[\mathrm{As}]$ substituted by $\mathrm{As}[\mathrm{Ga}]$ \\
\hline$(1 \times 1)-\zeta$ & (113) $[(113)]$ & as $(1 \times 1)-\beta$ but 2 nd layer As [Ga] substituted by $\mathrm{Ga}[\mathrm{As}]$ \\
\hline$(2 \times 1)-\alpha$ & $(112)[(112)]$ & as $(1 \times 1)-\alpha$ plus As $[\mathrm{Ga}]$ dimer formation along $[\overline{1} 10]$ \\
\hline $\mathrm{o}(2 \times 1)-\alpha$ & $(113)[(\overline{113})]$ & as $(1 \times 1)-\alpha$ plus As $[\mathrm{Ga}]$ dimer formation along [011] \\
\hline$(2 \times 1)-\beta$ & $\{112\}$ & as $(1 \times 1)-\beta$ plus $\mathrm{Ga}$ dimer formation along [111] \\
\hline $\mathrm{o}(2 \times 1)-\beta$ & $(113)[(\overline{113})]$ & as $\mathrm{o}(2 \times 1)-\alpha$ plus one $\mathrm{As}[\mathrm{Ga}]$ adatom \\
\hline $\mathrm{o}(8 \times 1)$ & $(113)$ & Ref. 13 \\
\hline
\end{tabular}


with $E_{\text {tot }}$ the total energy of the slab. $\mu_{i}$ and $N_{i}$ denote the free energy per particle and the number of particles in the slab of the species $i$, respectively. Chemical equilibrium requires that the sum of $\mu_{\mathrm{Ga}}$ and $\mu_{\mathrm{As}}$ equals $\mu_{\mathrm{GaAs}}$, the energy per Ga-As pair in bulk GaAs. Thus, there is only one independent variable describing the chemical environment, and we take $\mu_{\mathrm{As}}$ for it. Furthermore, this chemical potential should be defined in a particular range according to the condition that $\mu_{\mathrm{Ga}}$ and $\mu_{\mathrm{As}}$ have to be smaller than the chemical potential of any corresponding solid phase:

$$
\mu_{\mathrm{Ga}} \leqslant \mu_{\mathrm{Ga} \text { bulk }}, \quad \mu_{\mathrm{As}} \leqslant \mu_{\mathrm{As} \text { bulk }} .
$$

Similar calculations for the (100), (110), (111), and (1̄11) surfaces of GaAs ar described in Ref. 12, where more details can be found.

The results for the $\{112\}$ surfaces are summarized in Fig. 4. In order to investigate their stability we have calculated the surface energy for the faceted structures assembled according to Herring's construction combining the $\{110\}$, $\{111\},\{100\}$, and $\{113\}$ faces. From Fig. 4 one recognizes that faceting lowers the energy with respect to all considered surface structures. According to our calculations the (112) surface facets in agreement with experiments. Because of neglecting of higher index facets in the calculations (as $\{124\})$ the theoretically predicted roof arrangement consisting of (111) and (113) planes may not be the real stable structure, as seemingly confirmed by the disagreement with experiment. The occurrence of $\{124\}$ facets is an important fingerprint that this may be another so far unexplored lowenergy surface. An upper limit for $\gamma_{(124)}$ is estimated as follows: Using the geometrical condition

$$
\sum_{i=1}^{N} A_{i} \mathbf{n}_{i}=0
$$

with $A_{i}$ the area of the face, $N$ the number of considered facets, and $\mathbf{n}_{i}$ the versor normal to the $i$ th face, and the energy relation

$$
\gamma_{\mathbf{n}_{j}}=\sum_{\substack{i=1 \\ i \neq j}}^{N} \frac{A_{i}}{A_{j}} \gamma_{\mathbf{n}_{i}}
$$

one obtains:

$$
\gamma_{(124)} \leqslant \frac{5}{4} \sqrt{\frac{11}{21}} \gamma_{(113)}+\frac{1}{4 \sqrt{7}} \gamma_{(111)}=0.91 \gamma_{(113)}+0.09 \gamma_{(111)}
$$

and also

$$
\gamma_{(124)} \leqslant 5 \sqrt{\frac{2}{21}} \gamma_{(110)}+\frac{1}{\sqrt{7}} \gamma_{(111)}=1.54 \gamma_{(110)}-0.38 \gamma_{(111)} \text {. }
$$

The important and pivoting point of the theoretical study is the prediction of the faceting for the other surfaces. The (112) surface undergoes faceting as well. It exhibits roofs consisting of $\{110\}$ planes along the [111] direction under As-rich conditions and roofs consisting of (111) and (11) planes pointing along the $[01 \overline{1}]$ direction under Ga-rich conditions. From these results it is likely to interpret the experimentally observed structures (see Fig. 2, lower panel) as fac-
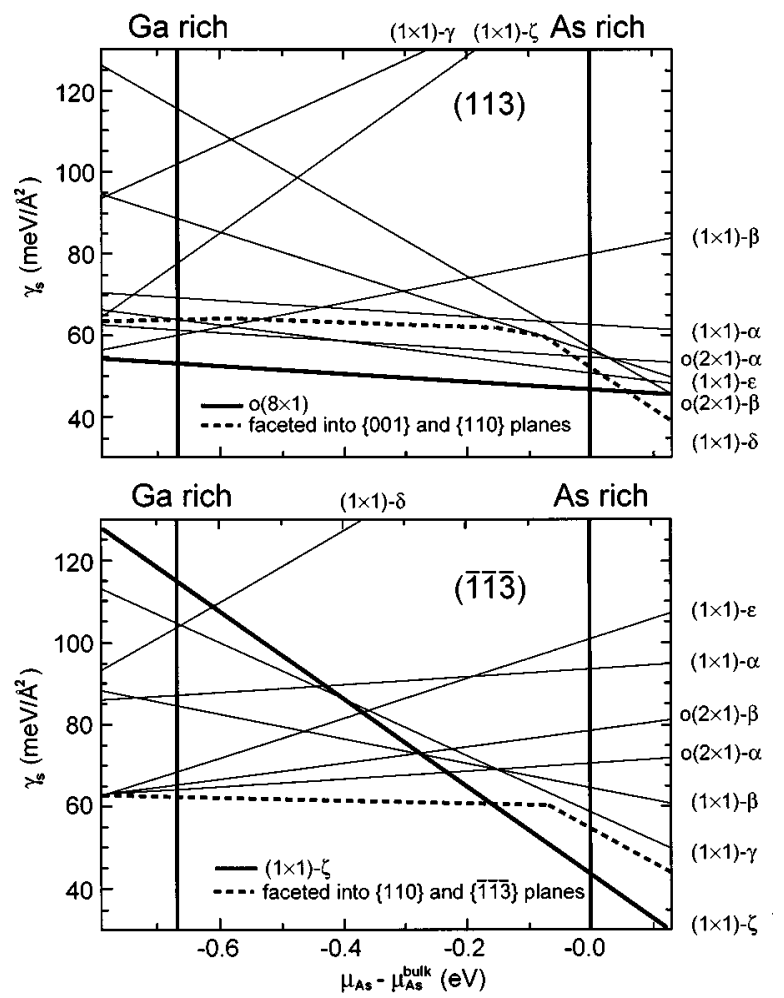

FIG. 5. Calculated surface energies of the GaAs (113) (upper part) and GaAs (113) surface (lower part) as a function of the As chemical potential. For the different models see Table I.

eted depressions terminated by all four faces as it could occur at the value of $\mu_{\mathrm{As}}$ where the two basic facetings have the same free energy.

For the $\{113\}$ surfaces we show the calculated surface energy curves in Fig. 5. An important point is to confirm that the model proposed by Wassermeier et al. ${ }^{13}$ on the basis of scanning tunneling microscopy (STM) pictures has a very low surface free energy and is the stable structure for a wide range of the chemical potential. It has a value of $47 \mathrm{meV} / \AA^{2}$ under As rich conditions and of $53 \mathrm{meV} / \AA^{2}$ under Ga-rich conditions. The calculations were performed also for a large variety of $1 \times 1$ and $2 \times 1$ reconstructions. ${ }^{16}$ Remarkably, none of the other structures are more stable than the $8 \times 1$ whose surface free energy is comparable with that of the nonpolar (110) surface $\left(\gamma=46 \mathrm{meV} / \AA^{2}\right) .{ }^{16}$ This low energy hinders faceting into the low-index surfaces, $\{110\},\{100\}$, and $\{111\}$. Moreover, as a consequence the (113) face contributes considerably to the equilibrium crystal shape. The same is true for the GaAs (113) surface if one considers As-rich growth conditions. For this surface a $1 \times 1$ stable structure is found which is bulk truncated, As terminated with second layer Ga atoms substituted by As. In the limits of very As-rich conditions the calculated value of the surface energy for this atomic arrangement $\left(43 \mathrm{meV} / \AA^{2}\right)$ is even lower than the one evaluated for the (110) surface. However, according to our results under Ga-rich conditions also the $(\overline{1} \overline{1})$ surface undergoes faceting into $\{110\}$ and $\{\overline{1} \overline{1} \overline{1}\}$ planes in agreement with the experimental findings (see Fig. 3 and its inset). 


\section{CONCLUSION}

In conclusion, it is found by ab initio total-energy calculations that the Wassermeier model for the GaAs(113) surface exhibits a very low surface energy which is comparable to those found for low-index surfaces. The conclusions drawn on the stability of GaAs $\{113\}$ surfaces are furthermore supported by the theoretical and experimental analysis of the GaAs $\{112\}$ surfaces. These surfaces are not stabilized by reconstructions of low energy but are faceted exposing $\{110\},\{111\}$, and $\{113\}$ planes. For the $\operatorname{GaAs}(112)$ surfaces $\{124\}$ faces have been observed. This points out that other-so far unexplored-high-index surfaces may also be of importance and have to be investigated in future work. Finally, the appearance of faceting under different preparation conditions opens new possibilities for the use of highindex GaAs surfaces as templates that dictate the arrangement of the assembled quantum dots. This latter aspect may open possibility for tuning the size distribution and the density of the quantum dots by acting on the substrate.

\section{ACKNOWLEDGMENTS}

We are grateful to P. Geng for technical help, and to N. Moll, E. Pehilke, L. Geelhaar, and J. Marquez for fruitful discussions. We thank Ch. Meyne, the group of Professor W. Richter, TU Berlin, for preparing the AFM picture and G. Weinberg for preparing the SEM image. The project was supported by the Bundesministerium für Forschung und Bildung under Grant No. 05622 EBA4 and by the Deutsche Forschungsgemeinschaft (Sonderforschungsbereich 296, Project Nos. A2 and A5).
${ }^{1}$ Y. Arakawa and H. Sakaki, Appl. Phys. Lett. 40, 939 (1982); M. Asada, M. Miyamoto, and Y. Suematsu, IEEE J. Quantum Electron. QE-24, 523 (1988).

${ }^{2}$ N. N. Ledentsov, in Proceedings of the 23rd International Conference on the Physics of Semiconductors, edited by M. Scheffler and R. Zimmermann (World Scientific, Singapore, 1996), p. 19.

${ }^{3}$ E. Pehlke, N. Moll, A. Kley, and M. Scheffler, Appl. Phys. A: Solids Surf. 65, 525 (1997); E. Pehlke, N. Moll, and M. Scheffler, in Proceedings of the 23rd International Conference on the Physics of Semiconductors, edited by M. Scheffler and R. Zimmermann (World Scientific, Singapore, 1996), p. 1301.

${ }^{4}$ C. W. Snyder, B. G. Orr, D. Kessler, and L. M. Sander, Phys. Rev. Lett. 66, 3032 (1991).

${ }^{5}$ D. Leonard, M. Krishnamurthy, C. M. Reaves, S. P. Denbaars, and P. M. Petroff, Appl. Phys. Lett. 63, 3203 (1993).

${ }^{6}$ J. M. Moison, F. Houzay, F. Barthe, L. Leprince, E. André, and O. Vatel, Appl. Phys. Lett. 64, 196 (1994).

${ }^{7}$ S. Ruvimov et al., Phys. Rev. B 51, 14766 (1995).

${ }^{8}$ V. Bressler-Hill, S. Varma, A. Lorke, B. Z. Nosho, P. M. Petroff, and W. H. Weinberg, Phys. Rev. Lett. 74, 3209 (1995).

${ }^{9}$ M. Grundmann et al., Phys. Rev. Lett. 74, 4043 (1995).

${ }^{10}$ P. M. Petroff and G. Medeiros-Ribeiro, MRS Bull. 21, 50 (1996).

${ }^{11} \mathrm{~L}$. Samuelson et al., in Proceedings of the 23rd International Conference on the Physics of Semiconductors, edited by M. Scheffler and R. Zimmermann (World Scientific, Singapore, 1996), p. 1269; B. Junno, T. Junno, M. S. Miller, and L. Samuelson, Appl. Phys. Lett. 72, 954 (1998).

${ }^{12}$ N. Moll, A. Kley, E. Pehlke, and M. Scheffler, Phys. Rev. B 54, 8844 (1996).

${ }^{13}$ M. Wassermeier, J. Sudijono, M. D. Johnson, K. T. Leung, B. G. Orr, L. Däweritz, and K. Ploog, Phys. Rev. B 51, 14721 (1995).

${ }^{14}$ R. Nötzel, N. N. Ledentsov, L. Däweritz, M. Hohenstein, and K. Ploog, Phys. Rev. Lett. 67, 3812 (1991).

${ }^{15}$ C. Setzer, J. Platen, P. Geng, W. Ranke, and K. Jacobi, Surf. Sci. 377379, 125 (1997).

${ }^{16} \mathrm{~A}$. Kley (unpublished). 